http://jmscr.igmpublication.org/home/

ISSN (e)-2347-176x ISSN (p) 2455-0450

crossref DOI: https://dx.doi.org/10.18535/jmscr/v8i12.36

Journal Of Medical Science And Clinical Research

IGM Publication

An Official Publication of IGM Publication

\title{
Acute Flaccid Quadriplegia as the Presenting Symptom of Sjogren's Syndrome- A Rare Presentation
}

\author{
Authors \\ Dr Sharan Krishna Menon ${ }^{1}$, Dr Pradeep Shenoy ${ }^{2}$ \\ ${ }^{1}$ Postgraduate, Department of General Medicine, KS Hegde Medical Academy, Mangalore \\ ${ }^{2}$ Assistant Professor, Department of Nephrology, KS Hegde Medical Academy, Mangalore
}

\begin{abstract}
Sjogren's syndrome is a slowly progressive autoimmune disorder characterized by lymphocytic infiltration of the exocrine glands resulting in xerostomia and dry eyes. The syndrome has unique features since it presents with a wide clinical spectrum from organ-specific autoimmune exocrinopathy to systemic disease. A small but significant number of these patients progress to develop malignant lymphoma. Middle-aged women (female-to-male ratio, 9:1) are primarily affected. However, Sjogren's syndrome may occur at any age, including childhood ${ }^{(1)}$. We present the case of a 42-year-old female who presented with flaccid paralysis of all four limbs and on evaluation was diagnosed to have Sjogren's syndrome. Primary Sjogren's Syndrome presenting as quadriplegia extremely rare and the significance of managing hypokalemiadue to renal tubular acidosis with potassium citrateinstead of potassium chloride is highlighted.

Keywords: Sjogren's syndrome, Hypokalemic periodic paralysis, distal renal tubular acidosis.
\end{abstract}

\section{Introduction}

One among the many causes of hypokalemia is distal renal tubular acidosis (dRTA), but it is rarely severe enough to present as hypokalaemic paralysis. A patient with Sjogren's syndrome, with no sicca symptoms, presenting for the first time with hypokalaemic paralysis due to dRTA is very uncommon ${ }^{(2)}$. Positive serology and salivary gland histopathologywas the only evidence of underlying Sjogren's syndrome in our patient.

\section{Case Report}

A 42-year-oldfemale patient with no known comorbidities was brought to the causality with complaints of acute onset weakness of bilateral upper and lower limb since 1 day. On presentation, her vitals were stable, BP:126/70mm Hg, PR: 86bpm, RR: 20 cycles/min, and oxygen saturation of $98 \%$ on room air. She had bilateral flaccid quadriplegia with diminished reflexes. Power was worse in the lower limb (MRC grade 1) than the upper limb (MRC grade 2). She had diminished deep tendon reflexes with bilateral plantar reflex mute. Sensory system and cranial nerve examinations did not reveal any abnormalities. All other systemic examinations were within normal limits. The patient is a known case of hypothyroidism for 3 years, for which she was on regular thyroid supplements. An MRI brain withwhole spine screening was done to rule out any neurological cause of quadriplegia, however, itwas reported to show only a diffuse 
disc bulge at C4-5 and C5-6 levels indenting the thecal sac with no significant disc bulge (Figure $1,2)$.

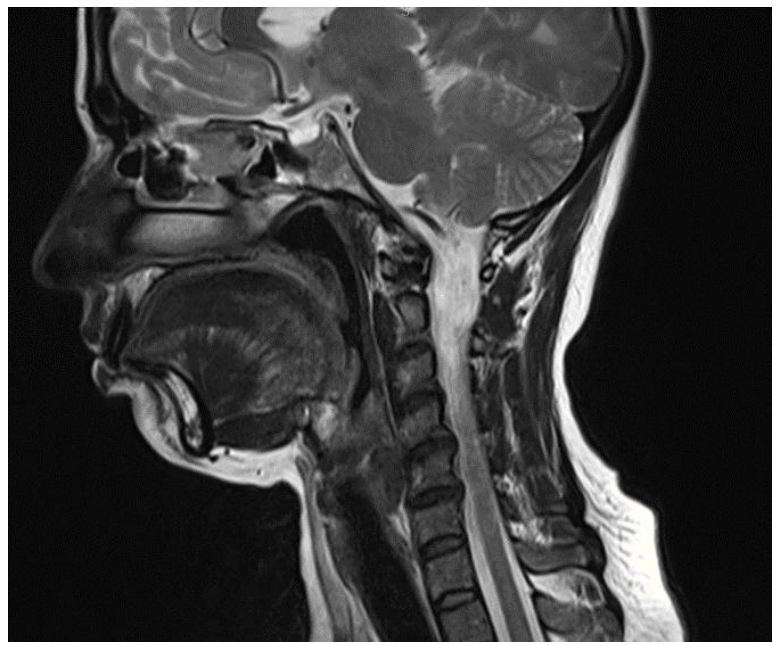

Figure 1: C5-6-disc bulge

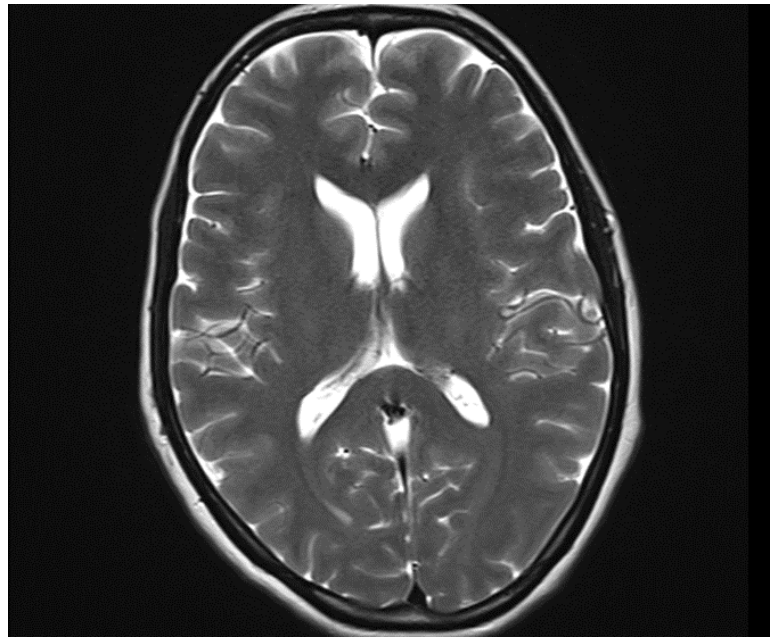

Figure 2: MRI brain normal study

On further evaluation, her blood reports showed a serum potassium level of $1.75 \mathrm{mmol} / \mathrm{L}$, and a diagnosis of hypokalemic periodic paralysis was made. Serum bicarbonate level was $6.1 \mathrm{mmol} / \mathrm{L}$ and urine $\mathrm{pH}$ was 6.0. Arterial blood gas analysis showed a pH of 7.25, pCO2 14, pO2 101, and bicarbonate of 10.2, indicative of severe metabolic acidosis. To identify the root cause of the metabolic acidosis, an ANA profile was done. The patient's ANA profile was positive for SS-A/Ro and SS-B/La suggestive of Primary Sjogren's syndrome.
ANA - Profile

(Serum)

Method : Line Immuno Assay(LIA)

\begin{tabular}{|c|c|c|}
\hline Autoantibody & Profile & Result \\
\hline dsDNA & \multirow{6}{*}{ SLE } & Negative \\
\hline Nucleosomes & & Negative \\
\hline Histones & & Negative \\
\hline $\mathrm{SmD1}$ & & Negative \\
\hline PCNA & & Negative \\
\hline $\mathrm{PO}$ & & Negative \\
\hline $\mathrm{SS}-\mathrm{A} / \mathrm{Ro} 60 \mathrm{Kd}$ & \multirow{3}{*}{ Sjogren Syndrome/SLE } & Positive \\
\hline $\mathrm{SS}-\mathrm{A} / \mathrm{Ro} 52 \mathrm{Kd}$ & & Positive \\
\hline SS-B/La & & Positive \\
\hline CENB-B & \multirow{2}{*}{ CREST/Scleroderma } & Negative \\
\hline Scl-70 & & Negative \\
\hline U1-snRNP & MCTD & Negative \\
\hline AMA M2 & PBC & Negative \\
\hline Jo-1 & \multirow{4}{*}{ Myositis } & Negative \\
\hline PM-Scl & & Negative \\
\hline $\mathrm{Mi}-2$ & & Negative \\
\hline $\mathrm{Ku}$ & & Negative \\
\hline
\end{tabular}

A lower lip mucosal biopsy of the minor salivary gland was done to confirm the diagnosis of Sjogren's syndrome. Histopathology was suggestive of Lymphocytic sialadenitis favoring the diagnosis of Sjogren's syndrome (Figure $3,4,5)$. A diagnosis of distal renal tubular acidosis secondary to Sjogren's syndrome was hence confirmed.

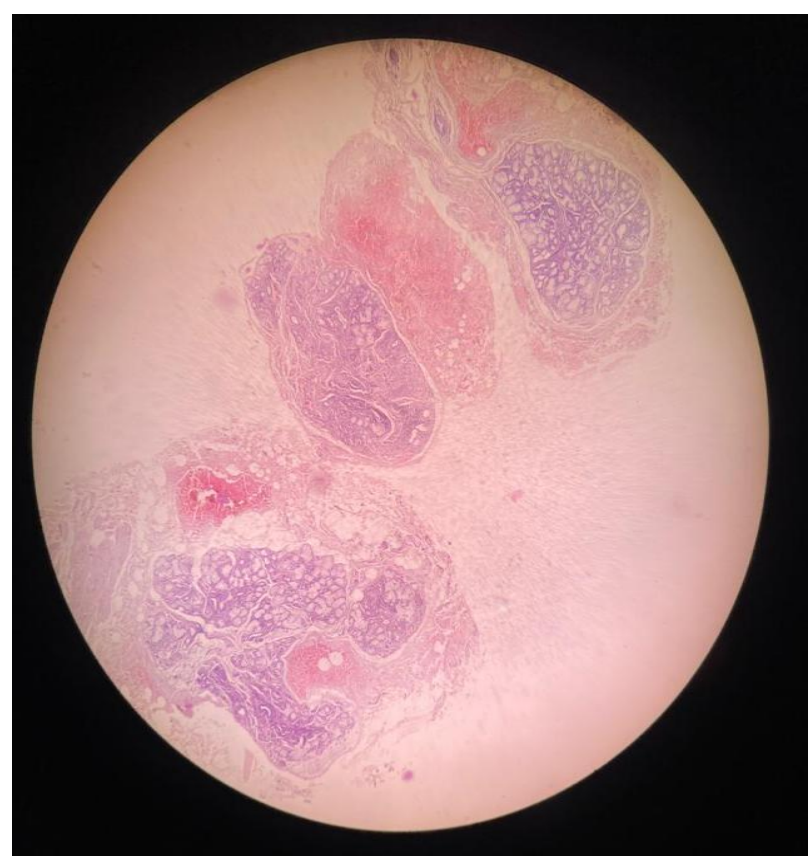

Figure 3: Low power view of salivary Acini with ducts and blood vessels 


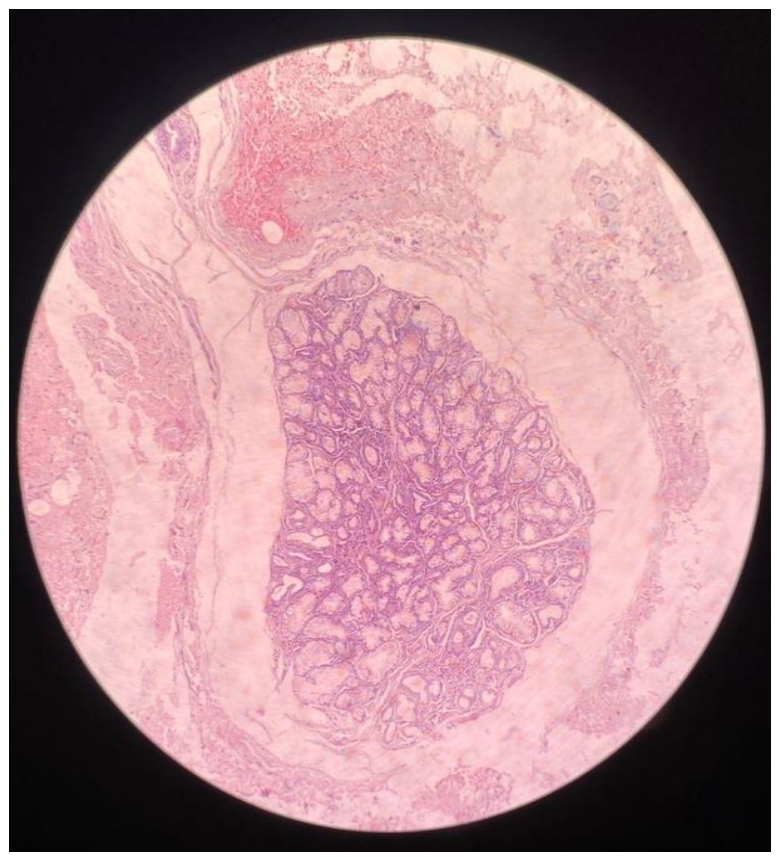

Figure 4: A salivary acini with ducts and blood vessels (40x H \& E)

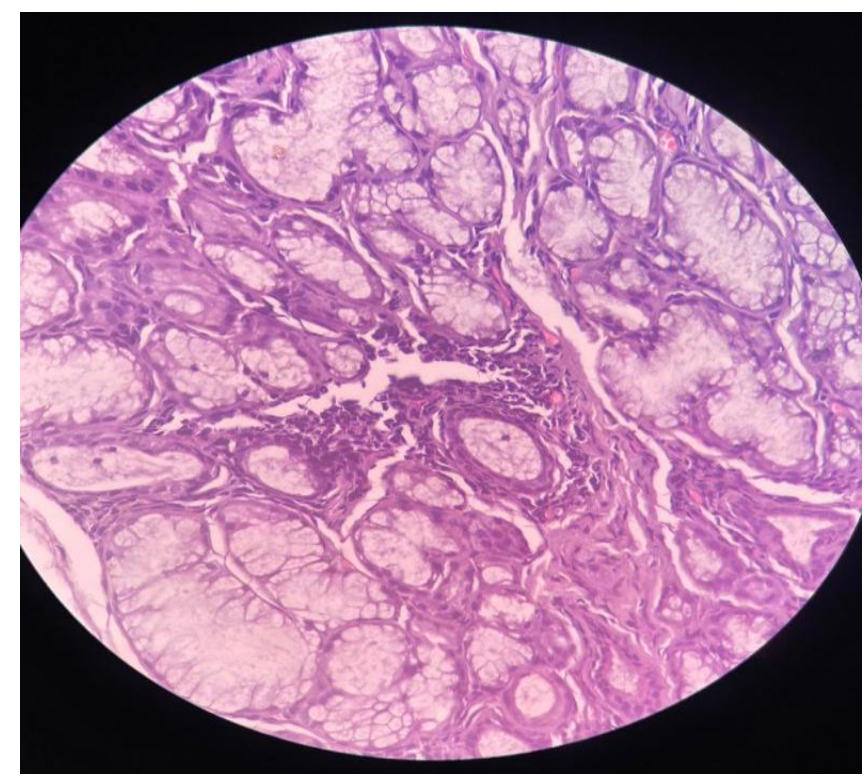

Figure 5: Higher power of the salivary lobule with lymphocytic infiltration

The patient was started on oral potassium citrate at a dose of $3 \mathrm{mmol} / \mathrm{kg} /$ body weight) and her muscle weakness improved over the next 4 days. Giventhe renal involvement, she was initiated on oral steroid therapy (oral prednisolone $1 \mathrm{mg} / \mathrm{kg}$ ) for four weeks and tapered slowly. Over a few weeks, the patient achieved normokalemia without oral potassium citrate therapy and there was complete remission of disease activity.

\section{Discussion}

Sjogren's syndrome (SS) is the second most common autoimmune disease affecting mainly middle-aged women. As a result of lymphocytic infiltration and destruction of salivary and lacrimal glands, the main symptom of the disease includes xerostomia and xerophthalmia ${ }^{(3)}$. The first and only manifestation of Sjogren's syndrome in our patient was hypokalemic paralysis due to dRTA. Her serology was strongly positive for Sjogren's syndrome and lip biopsy for salivary gland histopathology was suggestive of the same etiology, even though she had no sicca symptoms. This case brings light to the fact that Sjogren's needs to be considered in a case of dRTA even in the absence of classical manifestations of the disease.

Renal tubular acidosis (RTA) refers to a group of disorders characterized by defective renal acidbase regulation. The ability for normal urinary acidification is flawed, resulting in net acid retention and hyperchloremic metabolic $\operatorname{acidosis}^{(4)}$. Patients with Sjogren's syndrome with dRTA have interstitial nephritis with high levels of anti-carbonic anhydrase antibodies. These antibodies affect the function of carbonic anhydrase in cortical collecting ducts. The defect in acidificationis due to insufficient intact $\mathrm{H}^{+}$ATPase pumps in the intercalated cells ${ }^{(5)}$.

In our patient, daily life-long alkali replacement in a dose of $1-2 \mathrm{mEq} / \mathrm{kg}$ will prevent acute hypokalemia and the use of corticosteroids or other immunosuppressive drugs used for the primary disease have shown to reverse the renal tubular defect.

\section{Conflicts of Interest: Nil}

\section{References}

1. Kasper DL, Fauci AS, Hauser S, et al, editors. Harrison's principles of internal medicine, 19th ed. [monograph on the Internet]. New York: The McGraw-Hill Companies, Inc.; 2015 [cited 2017 Jan 4]. 
Available from:

http://www.accesspharmacy.com.

2. Muthukrishnan J, Dawra S, Marwaha V, Narayanan CS. Sjögren's syndrome presenting as hypokalemic paralysis. Med J Armed Forces India. 2015;71(Suppl 1):S172-S174. doi:10.1016/j.mjafi.2013.11.005.

3. Kaufman I, Schwartz D, Caspi D, Paran D. Sjögren's syndrome - not just Sicca: renal involvement in Sjögren's syndrome. Scand J Rheumatol. 2008 Jan;37(3):213-8.

4. Yaxley J, Pirrone C. Review of the Diagnostic Evaluation of Renal Tubular Acidosis. 2016;16(4):6.

5. Hopper J. Interstitial Nephritis in Sjogren's Syndrome. Intern Med. 1968;69(6):8. 